\title{
DEVELOPMENT AND EVALUATION OF TIOCONAZOLE LOADED EMULGEL
}

\author{
SHAILENDRA KUMAR SAH*, ASHUTOSH BADOLA, SAYANTAN MUKHOPADHYAY \\ Department of Pharmaceutics, Division of Pharmaceutical Sciences, Shri Guru Ram Rai Institute of Technology and Science, Uttarakhand \\ Technical University, Dehradun, 248001, Uttarakhand, India \\ Email: sahshailendra31@gmail.com \\ Received: 17 May 2017, Revised and Accepted: 22 Aug 2017
}

\begin{abstract}
Objective: The objective of the present work was to develop, characterize and evaluate the tioconazole loaded emulgel and to prove that emulgel can be the best alternative for delivery of hydrophobic drug topically.

Methods: for the preparation of stable Emulgel, firstly gels were prepared using different polymers as carbopol 934 and xanthan gum, followed by preparation of emulsions and finally mixed together (table 1). Emulgel was evaluated for physical appearance, $\mathrm{pH}$, spreadability, extrudability, viscosity, swelling index, dilution test, centrifuge test, drug content, in vitro release study, kinetic analysis of release data, antifungal activity and stability study for 3 mo. A comparative study was also performed between prepared emulgels with available marketed antifungal cream.

Results: All evaluation parameters were in acceptable range with good physical appearance and the pH in the range of 5.5 to 6.8 . The results show that the extrudability was in the range 15.63 to $35.27 \mathrm{~g} / \mathrm{cm}^{2}$; with spreadability in range of 6.6 to $8.833 \mathrm{~cm}$. swelling index of F3 was seen a maximum in $3 \mathrm{~h}$ of about $75.13 \%$. The viscosity was in the range of 15240 to $56340 \mathrm{cps}$ at 10 RPM. During in vitro release of all formulations, F1 and F5 showed a maximum in vitro drug release of $59.11 \%$ and $55.11 \%$ respectively in 8 h. The kinetic analysis of fitting the data in different model shows that the best formulation of F1 fits in the Higuchi model with regression coefficient ( $\left.\mathrm{R}^{2}\right)$ of 0.998 and show non-fickian diffusion. The formulations were found stable. F1 and F5 provide a similar zone of inhibition like to market cream.
\end{abstract}

Conclusion: Tioconazole emulgel provide the better platform for delivery of hydrophobic drug for topical route and so able to produce better patient compliance.

Keywords: Emulgel, Tioconazole, Hydrophobic drug, Topical drug delivery

(C) 2017 The Authors. Published by Innovare Academic Sciences Pvt Ltd. This is an open access article under the CC BY license (http://creativecommons.org/licenses/by/4.0/) DOI: http://dx.doi.org/10.22159/ijap.2017v9i5.20046

\section{INTRODUCTION}

Topical drug delivery is the potential route to deliver the drug producing low side effect in comparison with any other dosage forms. Drug concentration can be optimized to low concentration due to lack of metabolic elimination of drug before reaching the targeted site [1]. There are various conventional dosage forms for topical applications as cream, ointment, gel, but they shows fluctuation in bioavailability of drugs and are associated with other limitation as gel is limited to deliver the hydrophilic drugs, similarly ointment are limited for hydrophobic drug, so recently there have been novel approaches in the topical delivery to control the fluctuation of drug and deliver it in a controlled manner as per our need and to reduce the limitation associated with conventional preparation also to improve their properties for patient compliance [2]. The new approach in the field of topical drug delivery is introduced to deliver the hydrophobic drug that can enjoy the gelling property and whose rate can be controlled named Emulgel. It is simply where emulsion of the drug is prepared and that are incorporated in the gel [3].

Several antifungal agents are available in the market as cream, ointment, gel, powders, lotions etc. for dermatological preparations. Tioconazole is an antifungal agent of imidazole class which is used to treat infections caused by a fungus or yeast. Imidazoles having broad spectrum activity against dermatophytes, Candida and other fungi involved in deep mycosis, some gram-positive and anaerobic bacteria. Tioconazole has considerably greater fungicidal activity than clotrimazole, econazole, ketoconazole, and miconazole against Candida albicans and other Candida species. tioconazole is highly hydrophobic in nature that lies in Biopharmaceutical classification system (BCS)-II class [4, 5].

Taking above consideration, tioconazole was selected to formulate as emulgel, the aim of this study was to develop an emulgel formulation of tioconazole using two polymers, natural polymerxanthan gum and synthetic polymer-carbopol 934 in different concentration. The effect of using different polymer for gelling and its concentration variation on the release of the drug from the emulgels was investigated. Others parameters as physical properties, spreadability, rheological properties, extrudability, swelling index, antifungal activity of the emulgel were also evaluated and compared with the marketed cream of sertaconazole nitrate (Onabet ${ }^{\mathrm{TM}}$ ) of the same imidazole class antifungal to justify that the emulgel can be the alternative for cream or they can be better dosage form to deliver the drug than other conventional semi-solid preparations for hydrophobic drugs in topical treatment.

\section{MATERIALS AND METHODS}

\section{Materials}

Tioconazole was received as gift from Themis Medicare Pvt. Ltd. Haridwar (India). All other excipients were of analytical grade procured from Central drug house Pvt. Ltd. Delhi (India). Distilled water was used throughout the studies. Onabet TM cream [sertaconazole nitrate $2 \% \mathrm{w} / \mathrm{w}$ ] of Glenmark Pharmaceuticals Ltd was procured from Pharmacy shop.

\section{Methods}

Drug-polymer compatibility studies

Drug-polymer compatibility studies were done for selection of polymer by Fourier Transform Infrared spectroscopy (FTIR) [6].

\section{Infrared spectroscopy}

Infrared spectra were recorded in the region of 4000 to $400 \mathrm{~cm}^{-1}$ by Perkin Elmer Spectrum Two ${ }^{\mathrm{TM}}$ Spectrophotometer.

\section{Formulation and evaluation}

\section{Preparation of emulgel formulation in different polymer}

The composition of emulgel formulations is given in table 1. Emulgel was prepared in two steps, the first formation of emulsion than the 
addition of emulsion into the gel. In formulations F1, F2, F3 gel base were prepared by dispersing carbopol 934 in distilled water and the $\mathrm{pH}$ was adjusted to 6 to 6.5 using triethanolamine (TEA). In formulations F4, F5, F6 xanthan gum was dispersed in cold water to form a gel. Oil phase of the emulsion was prepared by heating liquid paraffin and cetosteryl alcohol to $70^{\circ} \mathrm{C}$ to $80^{\circ} \mathrm{C}$ and dissolving span80 into it, then isopropyl myristate was added to oil phase. The aqueous phase is prepared by dissolving tween-80 and addition of propylene glycol to it, further tioconazole dissolved in alcohol was added to same. Both the oil and aqueous phase was heated to $70^{\circ} \mathrm{C}$ to $80^{\circ} \mathrm{C}$ and then the aqueous phase was added to oil under constant stirring until the temperature reaches to room temperature. The obtained emulsion was then added to Gel under stirring in the ratio of $1: 1$ to obtain the Emulgel [7].

Table 1: Composition of tioconazole emulgel formulations

\begin{tabular}{|c|c|c|c|c|c|c|}
\hline \multirow[t]{2}{*}{ Ingredients (\%w/w) } & \multicolumn{6}{|c|}{ Formulations } \\
\hline & F1 & F2 & F3 & F4 & F5 & F6 \\
\hline Tioconazole & 1 & 1 & 1 & 1 & 1 & 1 \\
\hline Carbopol 934 & 0.5 & 1 & 1.5 & - & -- & - \\
\hline Xanthan gum & -- & -- & -- & 1 & 1.5 & 2 \\
\hline Light liquid paraffin & 7 & 7 & 7 & 7 & 7 & 7 \\
\hline Span 80 & 1 & 1 & 1 & 1 & 1 & 1 \\
\hline Cetosteryl alcohol & 3 & 3 & 3 & 3 & 3 & 3 \\
\hline Isopropyl Myristate & 7 & 7 & 7 & 7 & 7 & 7 \\
\hline Tween 80 & 1 & 1 & 1 & 1 & 1 & 1 \\
\hline Propylene glycol & 5 & 5 & 5 & 5 & 5 & 5 \\
\hline Ethanol & 2.5 & 2.5 & 2.5 & 2.5 & 2.5 & 2.5 \\
\hline Water & q. $s$ & q. $s$ & q. $s$ & q. $s$ & q. $s$ & q. $s$ \\
\hline
\end{tabular}

\section{Evaluations of emulgel and marketed cream}

\section{Physical appearance}

The prepared Emulgel and the marketed cream Onabet ${ }^{\mathrm{TM}}$ were inspected visually for their color, homogeneity, grittiness, phase separation [8].

\section{pH}

$\mathrm{pH}$ of $1 \%$ dispersion of Emulgel and the marketed cream Onabet ${ }^{\mathrm{TM}} \mathrm{in}$ distilled water was measured with digital $\mathrm{pH}$ meter. Measurement was done in triplicate and averages were calculated [8].

\section{Extrudability (tube test)}

Here the weight required extruding $0.5 \mathrm{~cm}$ ribbon of Emulgel and the marketed cream Onabet ${ }^{\mathrm{TM}}$ in $10 \mathrm{~s}$ from the lacquered collapsible aluminum tube is determined. The test was repeated and the average values were used for the calculation [8]

\section{Formula for extrudability calculation}

Extrudability $=$ weight applied to extrude emulgel from tube $(\mathrm{g})$ /Area $\left(\mathrm{cm}^{2}\right)$

\section{Swelling index}

$1 \mathrm{~g}$ of Emulgel formulations and the marketed cream Onabet ${ }^{\mathrm{TM}}$ was taken on porous aluminum foil and then placed in the petri dish containing $10 \mathrm{ml}$ distilled water. The samples were taken from the petri dish at a different time interval and left undisturbed in a dry place for some time so that the external liquid is removed and weighed. Swelling index is then calculated by using below formula [8].

$$
\text { Swelling Index }(\mathrm{SW}) \%=\left[\left(\mathrm{W}_{\mathrm{t}}-\mathrm{W}_{\mathrm{o}}\right) / \mathrm{W}_{\mathrm{o}}\right] \times 100
$$

Where $(\mathrm{SW}) \%$ = Equilibrium percent swelling,

$\mathrm{W}_{\mathrm{t}}=$ Weight of swollen emulgel after time $\mathrm{t}$,

$\mathrm{W}_{\mathrm{o}}=$ Original weight of emulgel at zero time

\section{Spreadability}

It expresses the extent of the area to which formulation readily spreads to the affected part on the application. The therapeutic efficacy of the product depends on its spreading value. For determination of spreadability, $1 \mathrm{~g}$ of Emulgel formulations and the marketed cream Onabet ${ }^{\mathrm{TM}}$ was placed within the circle of $1 \mathrm{~cm}$ diameter pre-marked on a glass plate of $20 \times 20 \mathrm{~cm}$, on which a second glass plate was placed. A $500 \mathrm{~g}$ weight was allowed to rest on the upper glass plate for $5 \mathrm{~min}$. the increase in the diameter due to gel spreading was noted [9].

\section{Drug content}

1\% dilution of Emulgel formulations was done with a suitable solvent and filtered through Whatman filter paper. $1 \mathrm{ml}$ filtrate is further diluted to $10 \mathrm{ml}$, then the sample was analyzed in UV spectrophotometer against blank that is solvent without drug and absorbance was noted. The absorbance was fitted in the line of equation obtained from the calibration curve to obtain the concentration of drug [10].

\section{Dilution test}

1\% aqueous dilution of Emulgel and the marketed cream Onabet ${ }^{\mathrm{TM}}$ was done by adding Continuous phase and visually checked for phase separation and clarity [11].

\section{Centrifuge test}

$6 \mathrm{~g}$ of Emulgel was taken in $10 \mathrm{ml}$ graduated centrifuge tubes and were subjected to spin at 4000 RPM for $10 \mathrm{~min}$. The sample was observed for any phase separation occurrence [12].

\section{Viscosity}

The viscosity of prepared emulgel and Onabet $\mathrm{TM}$ marketed cream was measured by Brookfield DVE viscometer (AMETEK) with spindle 64 . The preparation whose viscosity was to be determined was placed in the beaker and allowed to settle down at the room temperature. The spindle was then lowered down and rotated at 2, 5, 10, and $12 \mathrm{rpm}$. The viscosity in centipoises was observed and reported [13].

\section{In vitro drug release}

In vitro releases of emulgel were calculated out using Franz diffusion cell (effective diffusion area $4.9 \mathrm{~cm}^{2}$ and $14 \mathrm{ml}$ cell volume). Semi permeable egg membrane was clamped to the donor compartment and the receptor compartment was filled with phosphate buffer $\mathrm{pH}$ 5.5+methanol [9:1] which was the dissolution media. The temperature of the cell was maintained at $37 \pm 2{ }^{\circ} \mathrm{C}$. The medium was continuously stirred with the magnetic bead at $50 \mathrm{rpm}$. The emulgel $(500 \mathrm{mg}$ ) were spread uniformly on the membrane. The sample $1 \mathrm{ml}$ aliquots were withdrawn from the receptor at regular interval and replace with the equal amount of fresh dissolution media. The samples withdrawn were diluted to required and analyzed under UV-spectrophotometry. The cumulative percentage drug released were calculated with time [14].

\section{Kinetic analysis of drug release}

Kinetic analysis of the drug release was done by fitting the release data to the different release model given below to describe the proper release model: 


\section{Zero order release (cumulative \% drug release vs. time)}

$$
Q=K_{o} t
$$

Where $\mathrm{Q}$ is the amount of drug released at time $\mathrm{t}, \mathrm{K}_{0}$ is the zeroorder release rate.

\section{First order release (log cumulative \% drug retained vs. time)}

$$
\ln (100-Q)=\ln 100-K_{1} t
$$

Where $Q$ is the percent of drug released at time $t, K_{1}$ is the firstorder release rate constant.

\section{Higuchi model (cumulative \% drug retained vs. square root of} time)

$$
Q=K_{2} \sqrt{ } t
$$

Where $Q$ is the percent of drug released at time $t, K_{2}$ is the Higuchi square root of time release constant.

Korsmeyer-peppas model (log cumulative \% drug release v/s. log time)

$$
F=\left(\frac{M t}{M}\right)=K_{m} t^{n}
$$

Where $\mathrm{F}$ is fraction of drug released at time $\mathrm{t}, \mathrm{M}_{\mathrm{t}}$ is drug release at time $t, \mathrm{M}$ is the total amount of drug in dosage form, $\mathrm{K}_{\mathrm{m}}$ is a constant dependent on the geometry of the dosage form, $\mathrm{n}$ is diffusion exponent indicating the mechanism of drug release, if the value of $n$ is 0.5 it indicates fickian diffusion and if between 0.5 to 1 it is anomalous transport or Non-fickian diffusion [14].

\section{Antifungal activity}

$19.5 \mathrm{~g}$ of Potato dextrose agar (PDA) was transferred in a $500 \mathrm{ml}$ of conical flask and $500 \mathrm{ml}$ of purified water and heat is applied to dissolve completely. Sterilization was done for $15 \mathrm{~min}$ at $121^{\circ} \mathrm{C}$ at 15 lbs pressure in an autoclave. Agar well method was used to determine the zone of inhibition for antifungal activity of emulgel formulations. Aspergillus strain was obtained from the Department of Microbiology, self-cultured in SGRRITS, Dehradun. The medium was poured into the three petridish and cooled for few minutes at room temperature until it forms solidifies at room temperature and then the three cups are bored in each petridish with the help of sterile steel boarer of $6 \mathrm{~mm}$ and fungal strain was swabbed on the PDA plate and similar concentration of the standard formulation $(0.125 \mathrm{~g})$ and emulgel formulations $(0.25 \mathrm{~g}$ ) were placed in the bores with help of 18 gauges needles and the petri plates were incubated for 24 to $48 \mathrm{~h}$ at $25^{\circ} \mathrm{C}$ in incubators. The zone of inhibition was measured in $\mathrm{mm}$ [15].

\section{Stability studies}

The optimized formulation was subjected to accelerated stability for 3 mo as per ICH guidelines by keeping at $40+2{ }^{\circ} \mathrm{C} / 75+5 \% \mathrm{RH}$ and Samples were withdrawn on at a regular interval of 1, 2 and 3 mo for evaluation of physical appearance, $\mathrm{pH}$, viscosity, centrifuge test and drug content [16].

\section{RESULTS AND DISCUSSION}

\section{Drug-polymer compatibility studies}

FTIR study of pure Drug (fig. 1), Drug with carbopol 934 (fig. 2) and drug with xanthan gum (fig. 3) was performed. The evaluation was based on shifting of the fingerprint region as the experimental result shows there was no significant deviation between drug and drug with polymers which confirm the compatibility. So these polymers were selected for formulations [6].

\section{Evaluation of emulgel and marketed Cream}

\section{Physical appearance}

Physical appearances of the formulations are shown in fig. 4 and described in table 2 .

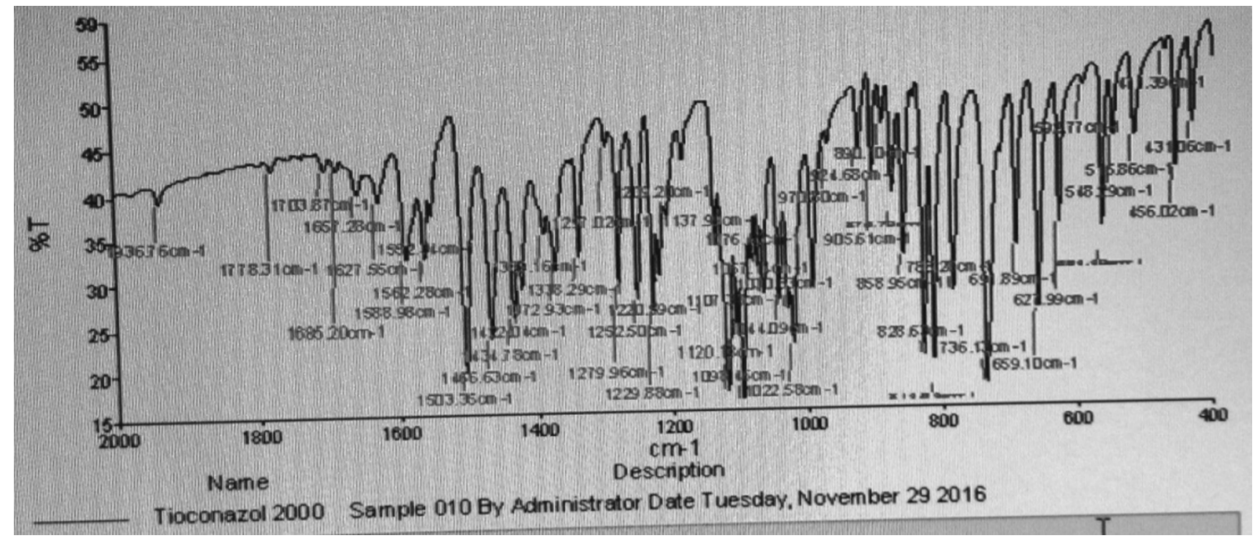

Fig. 1: FTIR of tioconazole sample

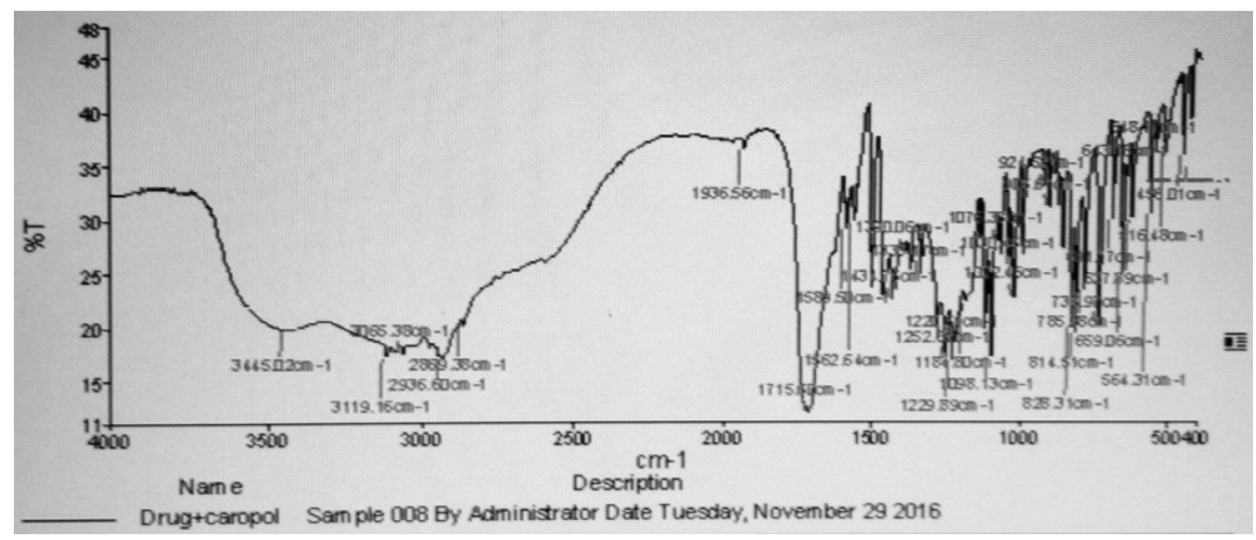

Fig. 2: FTIR-spectrum of tioconazole+carbopol 934 


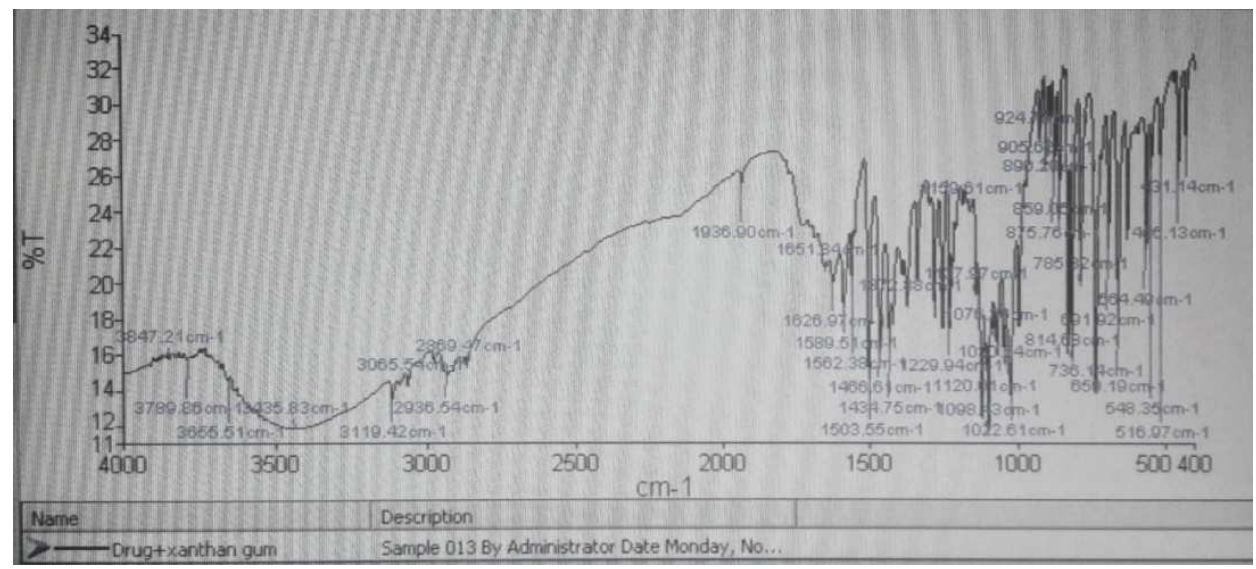

Fig. 3: FTIR spectrum of tioconazole+xanthan gum

Table 2: Physical characteristics

\begin{tabular}{llll}
\hline Formulation & Colour & Phase separation & Grittiness \\
\hline F1 & White & No & None \\
F2 & White & No & None \\
F3 & White & No & None \\
F4 & White & No & None \\
F5 & White & No & None \\
F6 & White & No & None \\
Onabet TM & White & No & None \\
\hline
\end{tabular}

$* n=3$, Average of three determinations

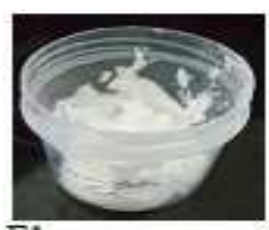

F1

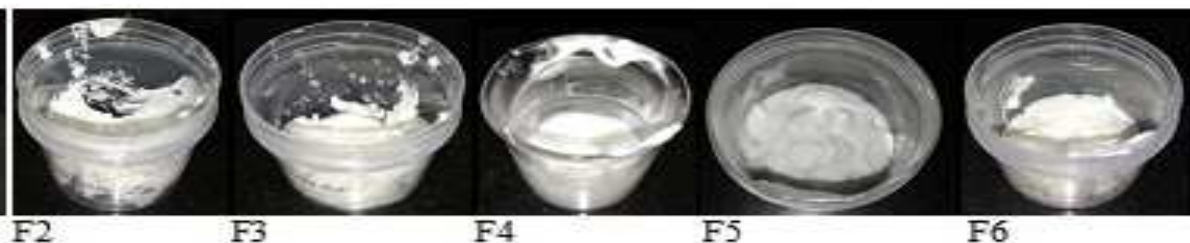

F6

Fig. 4: Different emulgel formulations

The formulations were white viscous creamy preparation with a smooth, homogeneous texture and glossy appearance with no grittiness and phase separation. That indicates the physical stability of the prepared formulations with acceptable features [7].
pH

$\mathrm{PH}^{\mathrm{H}}$ of different formulations is listed in table 3.

\section{Extrudability}

Extrudability of the different formulation is shown in table 4 .

Table 3: pH of emulgel formulation and marketed cream

\begin{tabular}{lllllll}
\hline Formulation & F1 & F2 & F3 & F4 & F5 & F6 \\
\hline $\mathrm{p}^{\mathrm{H}}$ & $5.733 \pm$ & $5.867 \pm$ & $5.733 \pm$ & $6.767 \pm$ & $6.733 \pm 0.057$ & $6.667 \pm$ \\
& 0.057 & 0.057 & 0.057 & 0.057 & & \\
\hline
\end{tabular}

${ }^{*} \mathrm{n}=3$, mean $\pm \mathrm{SD}$, The $\mathrm{p}^{\mathrm{H}}$ of the formulations was in the range 5.5 to 6.8 which match with the normal pH range of the skin and thus do not produce any skin irritation [1].

Table 4: Extrudability of emulgel formulations and marketed cream

\begin{tabular}{|c|c|c|c|c|c|c|c|}
\hline Formulation & F1 & F2 & F3 & F4 & F5 & F6 & Onabet TM \\
\hline Extrudability $\left(\mathrm{g} / \mathrm{cm}^{2}\right)$ & $20.67 \pm$ & $31.2 \pm$ & $35.27 \pm$ & $15.63 \pm$ & $17.8 \pm$ & $23.5 \pm$ & $19.8 \pm$ \\
\hline & 0.473 & 0.656 & 1.201 & 0.757 & 0.3 & 0.53 & 0.7 \\
\hline
\end{tabular}

$* n=3$, mean $\pm S D$

Formulation F1 and F4 with polymers carbopol 934 and xanthan gum respectively having low concentration among their respective formulations shows the low requirement of extrusion pressure and the extrudability rises with the increase in viscosity of the formulation. The formulation with xanthan gum requires low extrusion pressure in comparison with that of carbopol and all the 
results were acceptable in comparison to the marketed cream Onabet TM [2].

\section{Swelling index}

The swelling index of all formulations and marketed cream is represented in fig. 5 .

Formulations with carbopol 934 showed maximum swelling index in comparison with xanthan gum and were comparable to that of marketed cream. Among all the formulation, F3 emulgel with carbopol $934(1.5 \%)$ showed the highest swelling index. Variation in swelling index value may be dependent on the water uptake nature and chain strength of the polymer [1].

\section{Spreadability}

Spreadability of emulgel and marketed cream are shown in table 5.

Spreadability is carried out for all the formulations. It indicates how easily the emulgel will be spread on the affected area by the small amount of shear. Spreadability of emulgel formulation lies between .1-8.833. F1 shows good spreadibility among the carbopol polymer and F4 shows good spreadibility among the xanthan gum polymer [9].

\section{Drug content}

Drug content of prepared formulations are shown in table 6.

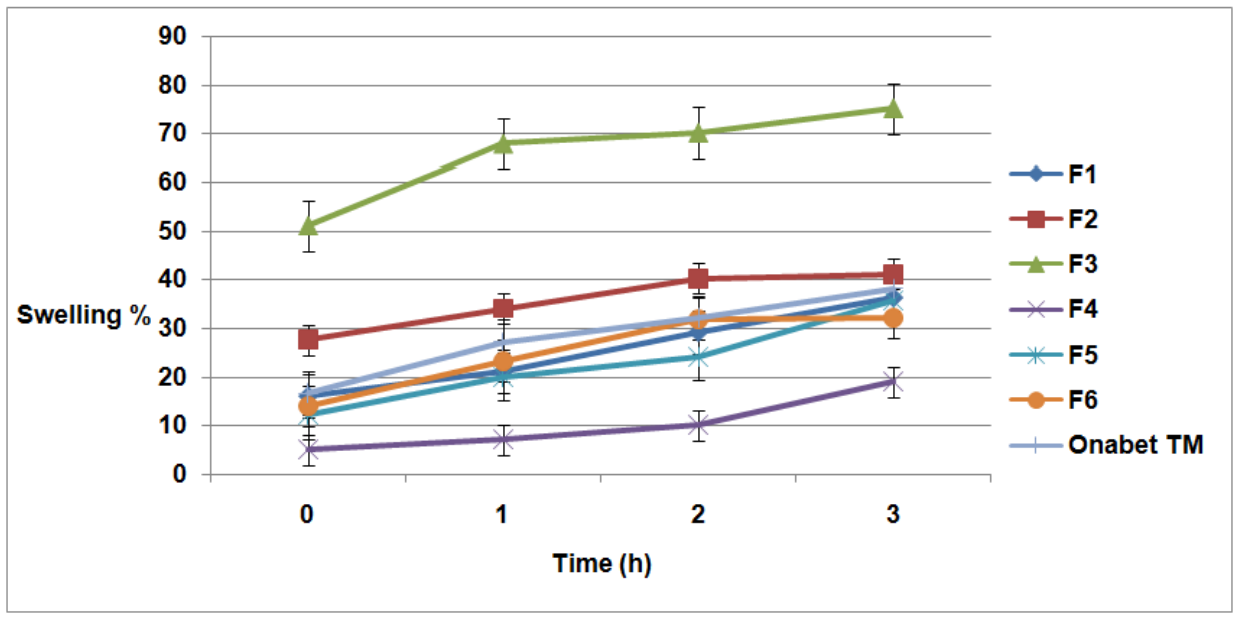

Fig. 5: \% swelling of formulated emulgels and marketed cream

Table 5: Spreadability of emulgel formulations and marketed cream

\begin{tabular}{llllllll}
\hline Formulation & F1 & F2 & F3 & F4 & F5 & F6 & Onabet TM \\
\hline Spreadability $(\mathrm{cm})$ & $6.6 \pm$ & $4.733 \pm$ & $4.1 \pm$ & $8.833 \pm$ & $8.433 \pm$ & $7.867 \pm$ & $6.767 \pm$ \\
& 0.1 & 0.115 & 0.1 & 0.153 & 0.115 & 0.115 & 0.057 \\
\hline
\end{tabular}

$* n=3$, mean \pm SD

Table 6: Drug content of emulgel formulations

\begin{tabular}{|c|c|c|c|c|c|c|}
\hline Formulation & F1 & F2 & F3 & F4 & F5 & F6 \\
\hline Drug content (\%) & $101.3 \pm$ & $98.27 \pm$ & $100.6 \pm$ & $97.95 \pm$ & $98.71 \pm$ & $95.64 \pm$ \\
\hline & 0.120 & 0.129 & 0.395 & 0.622 & 0.520 & 0.583 \\
\hline
\end{tabular}

$* \mathrm{n}=3$, mean $\pm \mathrm{SD}$

The drug content of the formulations is determined by UVspectrophotometer at a wavelength of $230 \mathrm{~nm}$, all the formulations shows the drug content in between the limit of $95 \%$ to $102 \%$ and there is no major effect of a change in polymers and its concentrations [10].

\section{Dilution test}

All prepared formulations and marketed cream were diluted with water and result indicated no phase separation upon dilution [11].

\section{Centrifuge test}

All prepared formulations and marketed cream showed no phase separation that indicates the stability of product on high shear rate [12].

\section{Viscosity}

The viscosity of emulgel formulation and marketed cream was determined using Brookfield Viscometer. Emulgel with carbopol 934 shows high viscosity in comparison to that of emulgel with xanthan gum. Results show the ascending viscosity of emulgel with an increase in the concentration of polymer in order $F 3>$ F2 $>$ F1 for carbopol 934, and a similar increase in viscosity was seen for the formulation with xanthan gum $\mathrm{F} 6>\mathrm{F} 5>\mathrm{F} 4$ as the concentration of polymer increases. Marketed cream Onabet TM shows viscosity comparable to emulgel formulation F1. Formulation shows decrease in viscosity with an increase in shear rate from 2 to 12 RPM that confirms the shear thinning properties of the formulations [13].

\section{In vitro drug release study}

Release of drug from the formulation was depended to the nature and concentration of polymer. Formulations with carbopol 934 shows the drug release in descending order $\mathrm{F} 1>\mathrm{F} 2>\mathrm{F} 3$ where the amount of drug released after $8 \mathrm{~h}$ was $59.11 \%, 40.77 \%, 35.18 \%$ respectively, and for the formulations with xanthan gum the drug release in descending order $\mathrm{F} 5>\mathrm{F} 4>\mathrm{F} 6$ where the amount of drug released after $8 \mathrm{~h}$ was $55.14 \%, 50.68 \%, 42.83 \%$ respectively. It has been concluded that the carbopol 934 emulgel with low concentration of polymer F1 shows maximum release. The release 
pattern with carbopol 934 emulgel was better than that of xanthan Gum. It shows carbopol 934 is better polymer than xanthan gum for formulating emulgel. Formulation F1 from carbopol polymer and formulation F5 from xanthan gum polymer shows the best result. The cumulative \% drug release profile of all the formulation batches has been shown in fig. 7 [16].

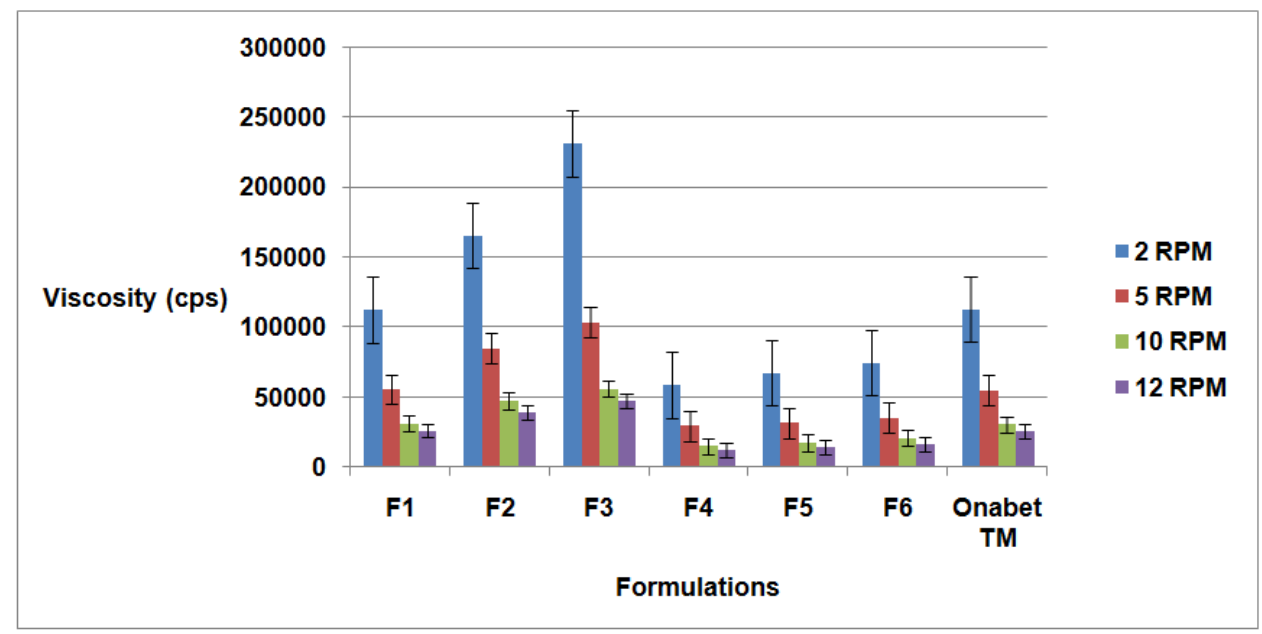

Fig. 6: Viscosity of emulgels and marketed cream with effects of shear rate (rpm) on it

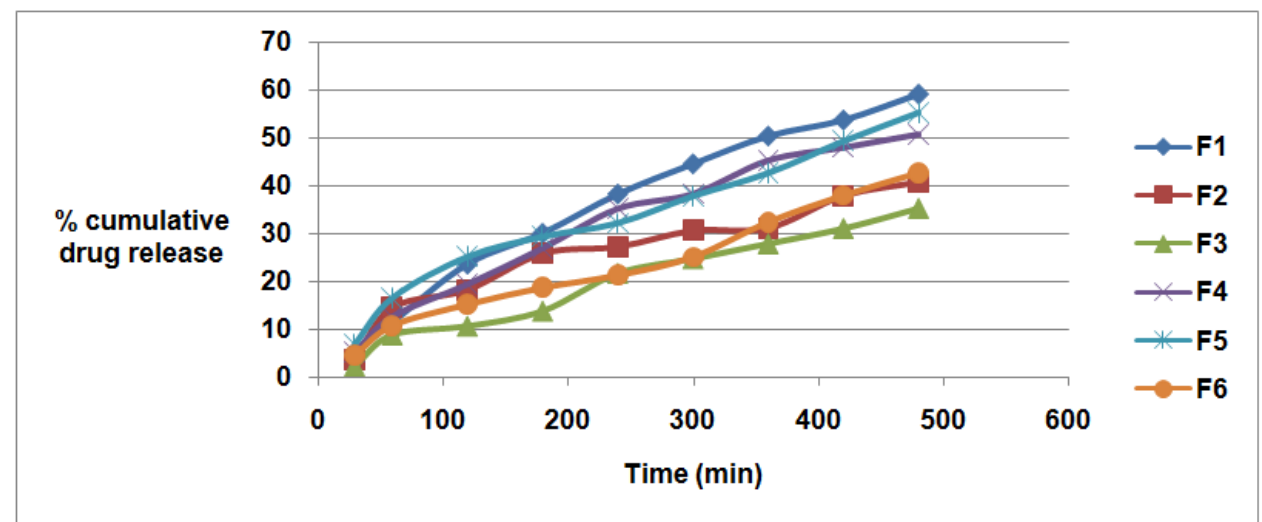

Fig. 7: In vitro cumulative \% drug release profile of emulgel formulations

\section{Kinetic analysis of drug release}

The results of In vitro release from formulations were plotted in different kinetic models like Zero order, First order, Higuchi plot and Korsmeyer Peppas plot and are represented in fig. 8, fig. 9, fig. 10, fig. 11 respectively. The regression coefficient value $R^{2}$ is reported for all formulations. The release data of best formulation F1 and F5 follows Higuchi model kinetics and first order kinetics respectively. All the formulation follows anomalous or non fickian diffusion for drug release. The regression coefficients of all formulations are listed in table 7 [16].

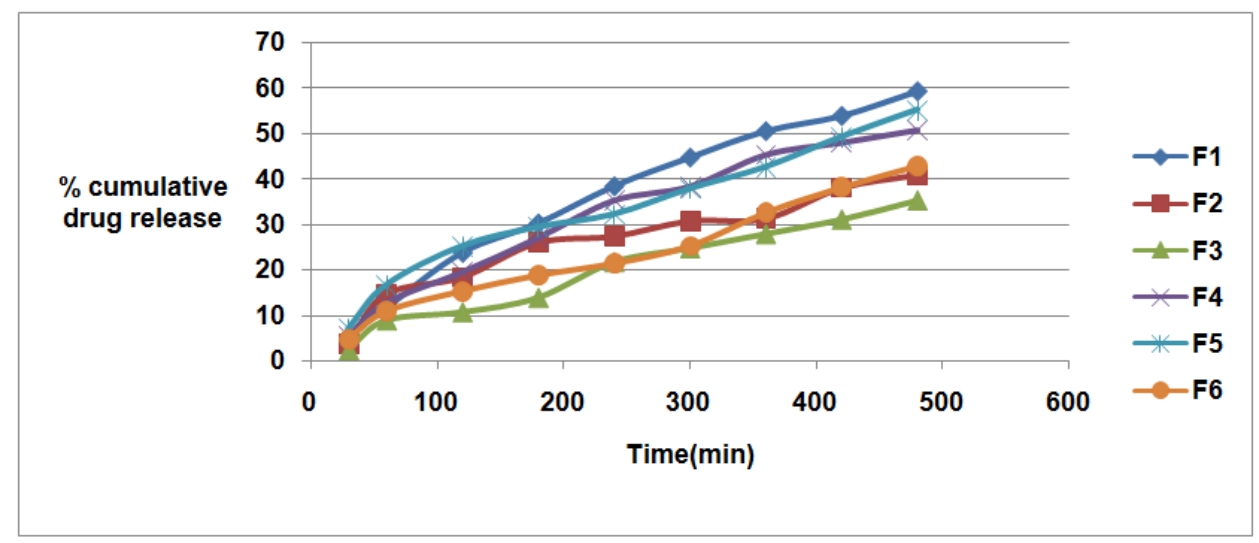

Fig. 8: Zero order release plot of emulgel formulations 


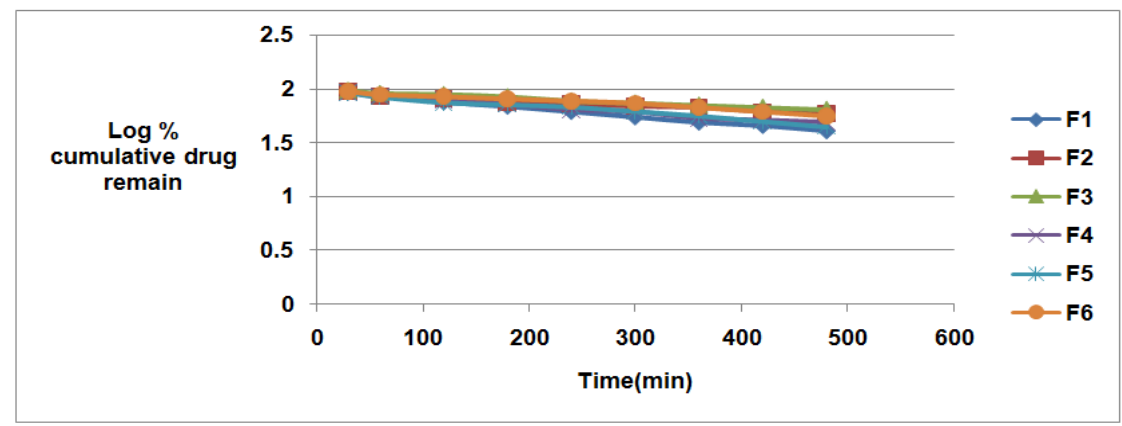

Fig. 9: First order release plot of emulgel formulations

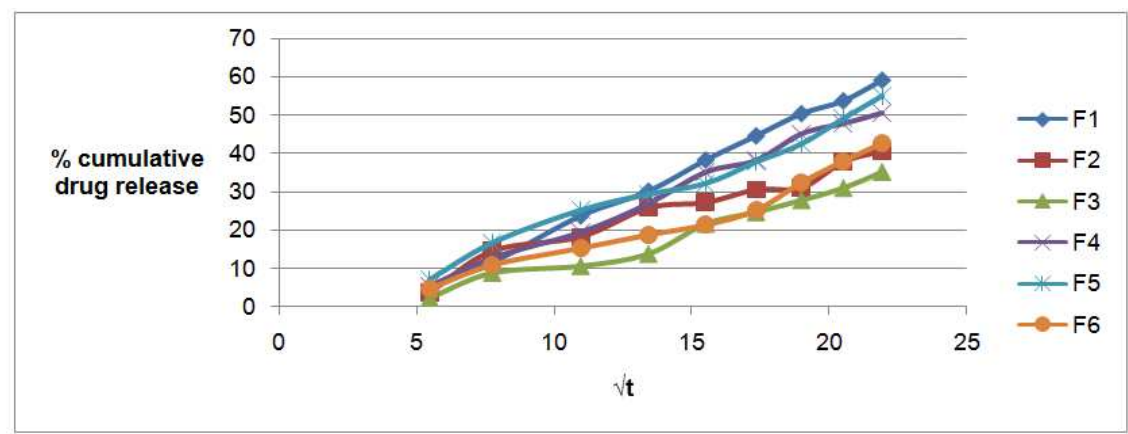

Fig. 10: Higuchi release plot of emulgel formulations

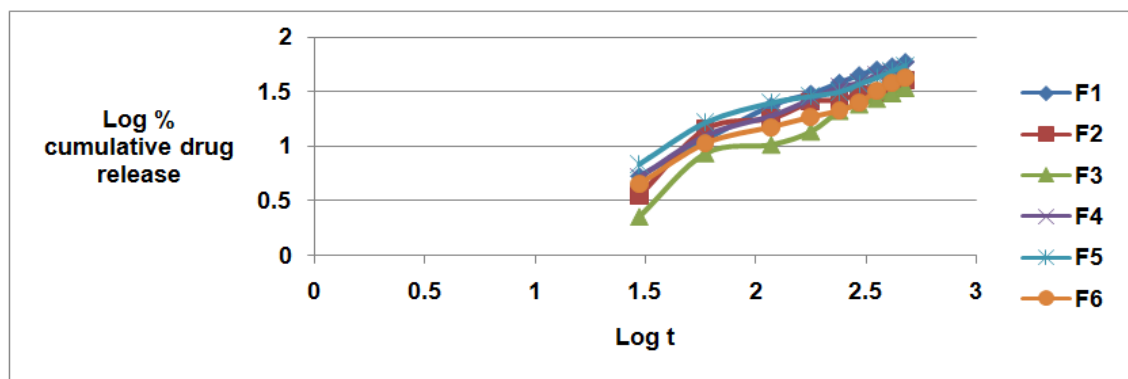

Fig. 11: Korsmeyer peppas release plot of emulgel formulations

Table 7: Release kinetic result of emulgel formulations

\begin{tabular}{|c|c|c|c|c|c|c|c|}
\hline \multirow[t]{2}{*}{ Formulation } & \multirow{2}{*}{$\begin{array}{l}\text { Zero order } \\
\mathbf{R}^{2}\end{array}$} & \multirow{2}{*}{$\begin{array}{l}\text { First order } \\
\mathbf{R}^{2}\end{array}$} & \multirow{2}{*}{$\begin{array}{l}\text { Higuchi plot } \\
\mathbf{R}^{2}\end{array}$} & \multicolumn{2}{|c|}{ Korsmeyer Peppas plot } & \multirow[t]{2}{*}{ Best fit model } & \multirow[t]{2}{*}{ mechanism of drug release } \\
\hline & & & & $\mathbf{R}^{2}$ & $\mathbf{n}$ & & \\
\hline F1 & 0.970 & 0.996 & 0.998 & 0.986 & 0.845 & Higuchi model & Non-fickian \\
\hline $\mathrm{F} 2$ & 0.918 & 0.957 & 0.964 & 0.890 & 0.746 & Higuchi model & Non-fickian \\
\hline F3 & 0.980 & 0.987 & 0.975 & 0.951 & 0.887 & First order & Non-fickian \\
\hline F4 & 0.966 & 0.987 & 0.995 & 0.983 & 0.787 & Higuchi model & Non-fickian \\
\hline F5 & 0.969 & 0.983 & 0.980 & 0.961 & 0.664 & First order & Non-fickian \\
\hline F6 & 0.983 & 0.977 & 0.953 & 0.973 & 0.730 & Zero order & Non-fickian \\
\hline
\end{tabular}

\section{Antifungal activity}

The antifungal activity of best two formulations F1 and F5 were seen and compared with the Onabet ${ }^{\mathrm{TM}}$ marketed cream (S) by measuring the zone of inhibition. The Formulation F1 shows a maximum zone of inhibition $18 \mathrm{~mm}$, whereas $\mathrm{F} 5$ and S shows the zone of inhibition of 14.67 and 17.33 respectively. The result indicates that the prepared emulgel F1 and F5 had a comparable antifungal activity to that of marketed cream (S) [17].

Table 10: Zone of inhibition of emulgel formulation and marketed cream

\begin{tabular}{ll}
\hline Formulation & Zone of Inhibition(mm) \\
\hline F1 & $18 \pm 1.732$ \\
F5 & $14.67 \pm 0.577$ \\
S (Onabet ${ }^{\text {TM }}$ cream) & $17.33 \pm 1.155$ \\
\hline
\end{tabular}

${ }^{*} \mathrm{n}=3$, mean $\pm \mathrm{SD}$ 


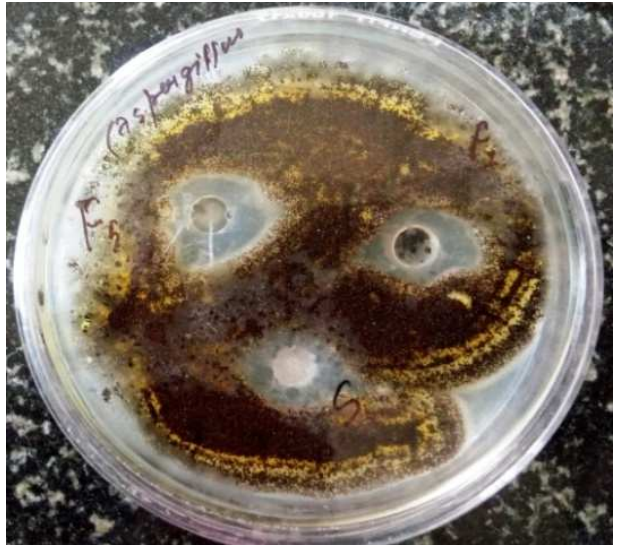

Fig. 12: Showing zone of inhibition in formulation F1, F5and marketed preparation $S$

\section{Stability studies}

The optimized formulations F1 and F5 with acceptable drug release were observed in stability study, for $3 \mathrm{mo}$ and all the parameters as physical appearance, $\mathrm{pH}$, viscosity and drug content was found to be stable upon storage for $3 \mathrm{mo}$, no major changes were observed in their parameters, the slight changes were seen which was in acceptable limit that indicates, the formulations are stable whose shelf life can be predicted [16].

\section{CONCLUSION}

Tioconazole Emulgel was formulated and compared with marketed cream and proved that emulgel can be, the better option than conventional as it minimizes the disadvantages associated with conventional semi-solid increasing the patient compatibility. The different gelling agent can be utilized such as carbopol 934 and xanthan gum. It can be novel approach to deliver the hydrophobic drug topically. It utilizes the two methods of formulating the emulsion and gel that facilitate to control the release of drugs. Various drugs can be formulated as emulgel and mostly the BCS class-II is efficient.

From the result and discussion, it is concluded that the emulgel with different polymer was in the acceptable range, showing the acceptable result of physical appearance, $\mathrm{pH}$, spreadability, extrudability, viscosity, swelling index, centrifuge test, dilution test, in vitro-release, antifungal activity and the formulation was found stable. However, among all formulations, F1 and F5 shows better result and concluded that tioconazole emulgel can be used for topical drug delivery with the aim of better efficacy. Among the polymer selection, the formulation with carbopol 934 with low concentration emulgel has better performances in comparison to xanthan gum. From the in vitro drug release studies, formulation F1 with carbopol 934 and formulation F5 with xanthan gum showed the maximum drug release of $55.91 \%$ and $55.14 \%$ respectively in 8 $\mathrm{h}$ with good physical properties. From the stability studies, after storage, the F1 and F5 formulation was observed in a stable form. Thus the tioconazole emulgel can be the best approach for topical delivery.
Recently only fewer drugs are being marketed as emulgel so there is open scope to utilize the emulgel as drug delivery system. Being simple to formulate they can be widely accepted by Researcher.

\section{CONFLICTS OF INTERESTS}

Declare none

\section{REFERENCES}

1. Ambala R, Vemula SK. Formulation and characterization of ketoprofen emulgels. J Appl Pharm Sci 2015;5:112-7.

2. Pednekar A, Dandagi P, Gadad A, Mastiholimath V. Formulation and characterisation of meloxicam loaded emulgel for topical application. Int J Pharm Pharm Sci 2015;7:216-22.

3. Ajazuddin, Alexander A, Khichariya A, Gupta S, Patel RJ, Giri TK, et al. Recent expansions in an emergent novel drug delivery technology: emulgel. J Controlled Release 2013;171:122-32.

4. Tripathi KD. Essentials of medical pharmacology. 6th ed. New Delhi: Jaypee publishers; 2008.

5. Clissold SP, Heel RC. Tioconazole a review of its antimicrobial activity and therapeutic use in superficial mycoses. Drugs 1986;31:29-51.

6. Rathnanand $\mathrm{M}$, Khatri SK, Nikhila R. Formulation and evaluation of wound healing activity of linezolid topical preparations on diabetic rats. Int J Appl Pharm 2016;8:30-6.

7. Mohamed MI. Optimization of chlorphenesin emulgel formulation. AAPS J 2004;6:1-7.

8. Kumar V, Antil M, Singh J, Kumar D. Emulgel-novel topical drug delivery system-a comprehensive review. Int J Pharm Sci Res 2016; 7:4733-42.

9. Khan WA, Kotta S, Ansari SA, Sharma RK, Kumar A, Ali J. Formulation development, optimization and evaluation of aloe vera gel for wound healing. Pharmacogn Mag 2013;9:6-10.

10. Khambete H, Deveda P, Jain A, Vyas N, Jai S. Gellified emulsion for sustain delivery of itraconazole for topical fungal diseases. Int J Pharm Pharm Sci 2010;2:104-12.

11. Ashara KC, Paun JS, Soniwala MM, Chavada JR, Mori NM. Microemulsion based emulgel: a novel topical drug delivery system. Asian Pac J Trop Dis 2014;4:27-32.

12. Kundan PJ, Laxman DS, Eknath PK. Stability evaluation of topical ointment comprising calcipotriol and prednicarbate. Int Res J Pharm 2015;6:43-7.

13. Sundari PP, Mullapudi D, Srinivas P. Formulation and evaluation of anti-arthritic poly herbal emulgel. Int J Pharm Technol 2014;6:6608-21.

14. Saxena A, Singh R. A review on levofloxacin in situ-gel formulation. Asian J Pharm Clin Res 2015;8:37-41.

15. Ali-Shtayeh MS, Abu Ghdeib SI. Antifungal activity of plant extracts against dermatophytes. Mycoses 1999;42:665-72.

16. Mulye SP, Wadkar KA, Kondawar MS. Formulation development and evaluation of Indomethacin emulgel. Pharm Sinica 2013;4:31-45.

17. Ghodekar SV, Chaudhari SP, Ratnaparakhi MP. Development and characterization of silver sulfadiazine emulgel for topical drug delivery. Int J Pharm Pharm Sci 2012;4:305-16.

\section{How to cite this article}

- Shailendra Kumar Sah, Ashutosh Badola, Sayantan Mukhopadhyay. Development and evaluation of tioconazole loaded emulgel. Int J Appl Pharm 2017;9(5):83-90. 